\title{
Future prospects for dry etching
}

\author{
Keizo Suzuki and Naoshi Itabashi \\ Central Research Laboratory, Hitachi Ltd. \\ 1-280 Higashi-Koigakubo, Kokubunji, Tokyo 185, Japan
}

\begin{abstract}
An overview of plasma etching, including its basic mechanism, its history, and the problem of trade-offs in its properties, is given as background for a proposal concerning the direction that research aimed at developing future dryetching techniques should take. Considering the three-level structure of the chemical, physical, and apparatus parameters of plasma etching, we need to develop new plasma sources with high controllability of the physical parameters. The ideal style is a soft three-dimensionally uniform plasma created using a waveplasma coupling power supply. Also, as device integration continues to advance and special requirements for new-concept devices arise, it may also be necessary to examine processes using post-plasma techniques, where minimizing damage will be especially important.
\end{abstract}

\section{Introduction}

The integration required for semiconductor-memory devices has approached the giga-bit order in recent years and the scale of fabrication will soon reach the $0.1-\mu \mathrm{m}$ order. Dry etching, like lithography, is a key technology for fine-pattern delineation. It now has two possible courses of future development. One is to improve current plasma etching techniques, and the other is to stop using plasma and develop new concepts of etching methodology. In this paper, we investigate the future prospects of dry etching, mainly focusing on plasma etching. First, we explain the role of plasma etching in the device fabrication process in terms of the basic mechanism of plasma etching and the desired performance. Next, the essential difficulty in plasma-etching development is discussed from the viewpoint of the structure of etching parameters. In this context, we review the history of plasma etching, mainly at the beginning stages of the research. Then, we propose what we feel are the most feasible future trends in plasma etching, including some important concepts for developing new plasma formation techniques. Finally, the importance and desired aspects of post-plasma etching are briefly discussed.

\section{Plasma Etching and the Desired Performance}

Figure 1 shows an etching process in LSI (Large-Scale Integrated circuit) fabrication. Etching is the process of projecting a mask pattern, prepared by lithography, onto a substrate surface by removing an objective material only at the open areas of the pattern without removing other materials. In the process, the following four requirements must be satisfied. First, fine-pattern delineation must be achieved, namely etching anisotropy. Erosion of the materials under the mask, which is called undercutting, must be avoided to use the surface area of the substrate efficiently. Second, a high etching rate is required to minimize the LSI cost. Third, high selectivity for different materials is needed to ensure that only the objective material is etched while the mask and end materials are not. Forth, there must be little damage or contamination to produce LSIs with high performance and reliability.

The basic mechanism of plasma etching is shown in Fig. 2, taking silicon etching using CF4 plasma as an example. The $\mathrm{CF}_{4}$ molecules are dissociated into smaller fragments; that is, neutral radicals or ions, such as $\mathrm{CF}_{\mathbf{X}}, \mathrm{F}, \mathrm{CF}_{\mathbf{X}}{ }^{+}$, and $\mathrm{F}^{+}$. In addition, neutral radicals, molecules, and ions that are larger than the parent $\mathrm{CF}_{4}$ molecules will be made in the plasma through subsequent gas-phase reactions. The radicals and ions thus generated strike the silicon-substrate surface to make volatile products, typically SiF4, and then the products evaporate from the surface. The radicals strike the surface in almost isotropic directions. Therefore, if etching proceeds only through reactions by the radicals, the etching will become isotropic, causing a large amount cf undercutting. On the other hand, ions are accelerated in the ion sheath formed above the surface, and strike the surface perpendicularly. Etching will therefore become anisotropic if the etching process includes the effect of the ions (1). The degree of ion assistance in the etching reaction is thus an important parameter in determining the anisotropy of etching. If the mean free path of the ions becomes shorter than the width of the ion sheath, the ions will collide with the other gas particles in the ion sheath. In that case, some of the ions cannot strike the surface perpendicularly, which decreases the anisotropy. Gas pressure is therefore another important parameter in the anisotropy of etching, since the mean free path of the ions depends on the pressure. 


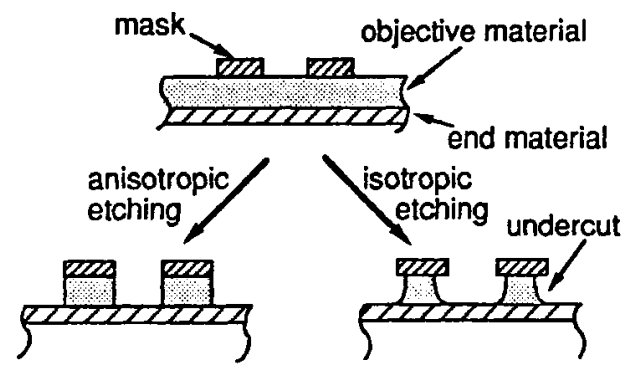

Desired Performance

- fine-pattern delineation (anisotropy)
- high etching rate
- low damage (low contamination)

Fig. 1 Etching process in LSI fabrication.



Typical Reaction (Ion-Assisted Reaction)

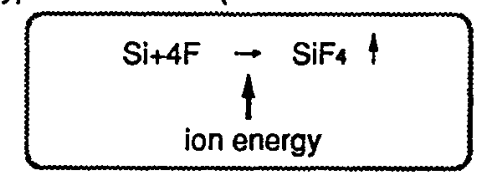

Fig. 2 Basic mechanism of plasma etching.

Side-wall protection (2) is also an important factor in fine-pattern delineation. For example, when silicon is etched with a $\mathrm{CF}_{4}+\mathrm{O}_{2}$ plasma, the silicon-substrate surface is covered by surface-reaction products, such as $\mathrm{Si}_{1} \mathrm{C}_{\mathrm{m}} \mathrm{F}_{\mathrm{n}}$ and $\mathrm{SiO}_{\mathrm{x}}$. The bottom of the etched pattern is irradiated with ions so that the reaction-product thin-film layer is removed through ion-assisted reactions and the bottom can be etched. On the other hand, the side wall of the pattern continues to be covered by the protective thin film since there are no incident ions, so the side wall cannot be etched.

\section{Difficulty in Plasma-Etching Development}

Trade-off relationships exist between the four requirements in plasma etching described in $\S 2$, as shown in Fig. 3. For example, etching anisotropy can be achieved with a high incidence flux of ions and a low incidence flux of neutral radicals to a substrate surface. However, such a condition is not desirable for the other requirements. That is, the physical process when using energetic ions with a higher incidence flux will cause more damage in the substrate material and will also lower the selectivity. Also, the lower incidence flux of radicals will not provide a high etching rate. Etching parameters must be carefully controlled under such a trade-off relationship, but precise control is not easy in plasma etching. This is the main problem to overcome in plasma-etching research, and it is becoming more serious with the increase in device integration.

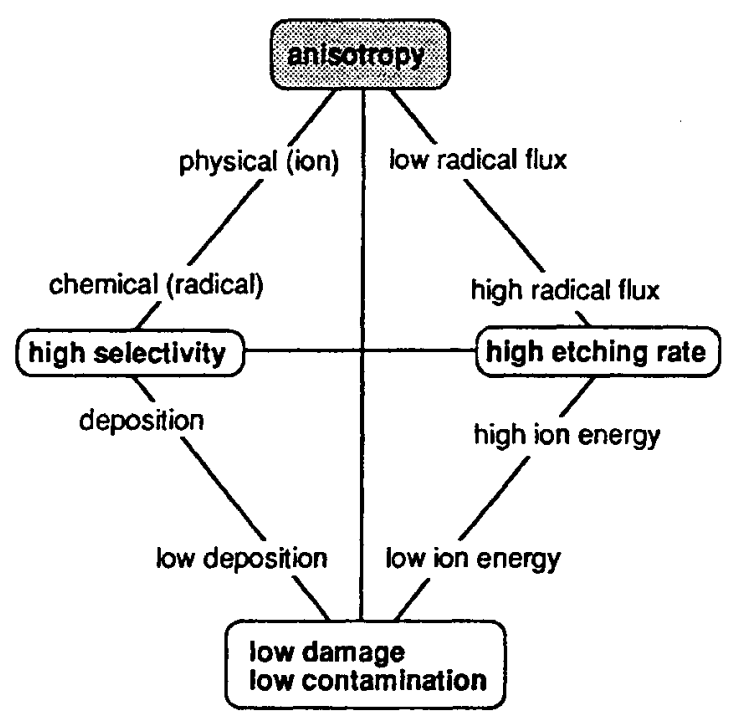

Fig. 3 Trade-off relationships in plasma etching.

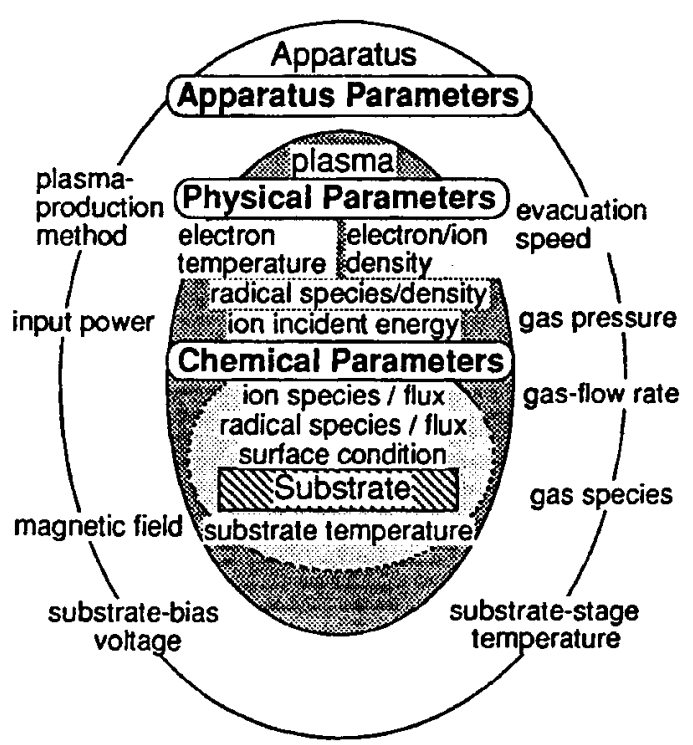

Fig. 4 Three-level structure of parameters in plasma etching. 
The difficulty will come from the three-level structure of parameters in plasma etching, as shown in Fig. 4. Since etching ultimately depends on the chemical reactions at the substrate surface, chemical parameters, such as substrate temperature, surface conditions, the incident radical species and their flux, and the incident ion species and their flux, are the most important for controlling the process. However, the chemical parameters depend somewhat on physical parameters, such as the electron temperature, the electron and ion densities, the radical species and their density, and the ion incident energy. However, even the physical parameters cannot be directly controlled by the operator. We can directly control only the apparatus parameters, such as the plasma-production method, the input power, the magnetic field, the substrate-bias voltage, the evacuation speed, the gas pressure, the gas-flow rate, the gas species, and the substrate-stage temperature. The three-level structure of the parameters is an essential point that distinguishes plasma etching from other dry-etching techniques, such as ion-beam etching and photoassisted etching, in which the physical parameters at least can be directly controlled by the operator.

\section{The Development of Plasma Etching}

The history of plasma etching is marked by the development of new apparatus parameters for controlling physical and/or chemical parameters, as summarized in Fig. 5. In the beginning stage, around 1975, the main effort was directed towards finding new apparatus parameters that could be used to control physical parameters. In other words, the objective was to develop new plasma-production methods, such as those using RF plasma, ECR plasma (3), and magnetron plasma. From about 1980, the research target has been to find new apparatus parameters that could be used to control chemical parameters. This research produced many new concepts, such as ion-assisted etching (1), side-wall protection (2), the RF-bias method (4), low-temperature etching (5), time-modulated etching (6), and high-gas-flow-rate etching (7). However, little effort seems to have been directed towards the physical parameters during the 1980s. Consequently, the technical level of the physical side has lagged behind the other areas. Now that we cannot overcome the present obstacles that prevent us from satisfying recent process requirements - at least, not by any effort on the chemical side, which has become apparent since 1990 - we must start to work again at finding new apparatus parameters that will let us control the physical parameters. This means we must develop new plasma-production methods.

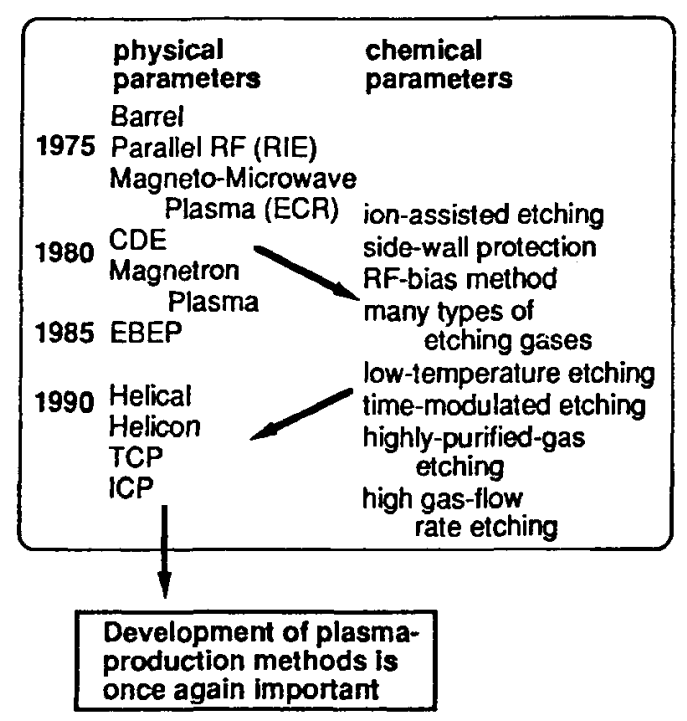

Fig. 5 The development of plasma etching.

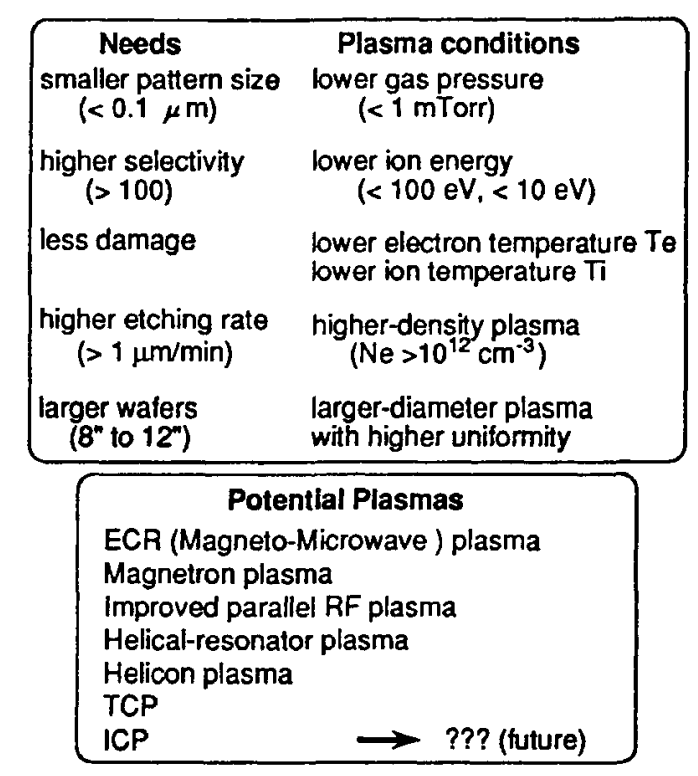

Fig. 6 Future trends in plasma etching.

\section{Future Trends in Plasma Etching}

The future trends in plasma etching will be shaped by the needs of future applications and the corresponding plasma conditions, as shown in Fig. 6. To delineate smaller patterns, a plasma at a lower gas-pressure is required. To achieve higher selectivity and less damage, a lower incident-ion energy and lower electron and ion temperatures are needed. To achieve a higher etching rate, a higher-density plasma is needed. To process larger wafers, a larger-diameter plasma with higher uniformity is needed. Some potential plasma sources, such as helical-resonator plasma (8), helicon plasma (9), TCP (10), and ICP (11), have been investigated with respect to these requirements. However, all of them are insufficient at the present stage, and further improvement is required. Important factors in the development of future plasma sources include the use of magnetic fields, the concept of a soft plasma, power supplies using wave-plasma coupling, source plasma and three-dimensionally-uniform plasma, and the control of the chamber-wall conditions. 


\subsection{The use of magnetic fields}

The advantages of using a magnetic field are the plasma confinement and the effective power injection, which make it easy to create a high-density plasma while operating at a low gas pressure, and controlling the electron-temperature over a wide range. On the other hand, disadvantages include localization of the plasma, which makes forming a highly-uniform plasma difficult, and resistance across the magnetic field, which gives rise to a plasma-potential distribution and, in certain cases, can cause damage. Also, since we cannot prevent the magnetic field crossing the chamber wall, contact between the high-density plasma and the chamber wall may contaminate the plasma. Moreover, the use of special instruments such as solenoid coils will increase the apparatus cost and size. However, these disadvantages do not appear to be insurmountable and will probably be overcome. Therefore, we should actively exploit the advantages to satisfy the future plasma requirements.

\subsection{Soft plasma}

Soft plasma means a plasma with low electron and ion temperatures. Since the ion motion is not significant in producing or sustaining a plasma, an $\mathrm{RF}$ power supply in the $\mathrm{kHz}$ or $\mathrm{MHz}$ frequency range, which accelerates the ions, is not appropriate. A microwave power supply in the GHz frequency range is better for plasma production. Generally, there is less interaction between the plasma and an electromagnetic field when the frequency of the power supply is higher. However, the use of a magnetic field lets us improve the power-supply efficiency. Therefore, a microwave power supply and a magnetic field will inevitably be used to produce soft plasma.

\subsection{Power supply using wave-plasma coupling}

If the electrodes that introduce the electro-magnetic field into the plasma are set up in a vacuum, they will cause contamination in the plasma. Therefore, here we will only talk about supplying the electromagnetic field through the chamber wall from the outside.

There are two ways to couple the plasma and the electro-magnetic field. One is quasi-static coupling and the other is wave-plasma coupling, as shown in Fig. 7. In the former, electrodes are set up slightly outside of the chamber wall. Then, a strong electro-magnetic field is created near the chamber wall where high-density plasma is produced. This causes plasma contamination from the chamber wall. On the other hand, in wave-plasma coupling, an electro-magnetic wave penetrates into the plasma through the insulator chamber wall, and interacts with the plasma in a particular spatial region to release the power into the plasma. In this method, a high-density plasma with very little contamination is produced away from the chamber wall. Therefore, to make soft clean plasma, wave-plasma coupling appears to be more promising than quasi-static coupling.

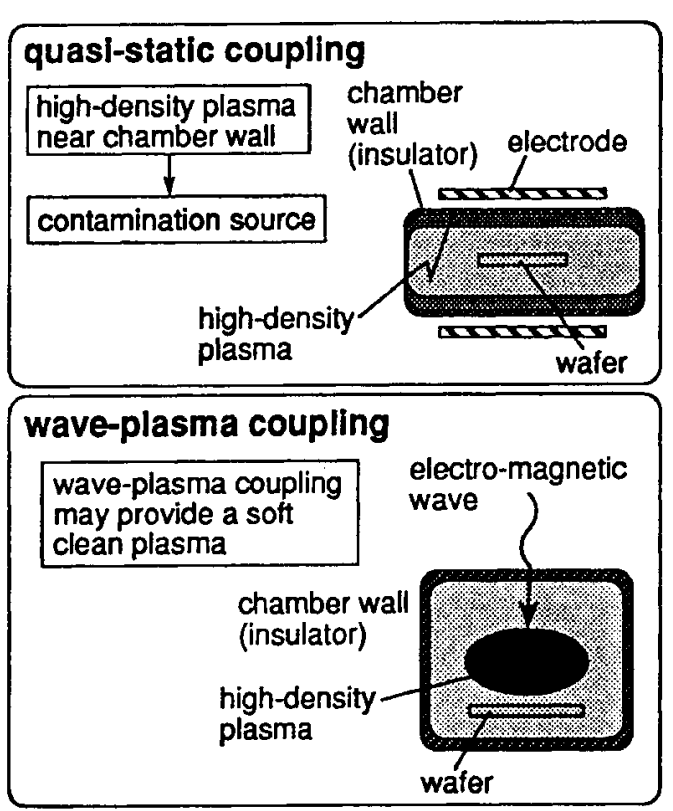

Fig. 7 Coupling between a plasma and an electro-magnetic field.

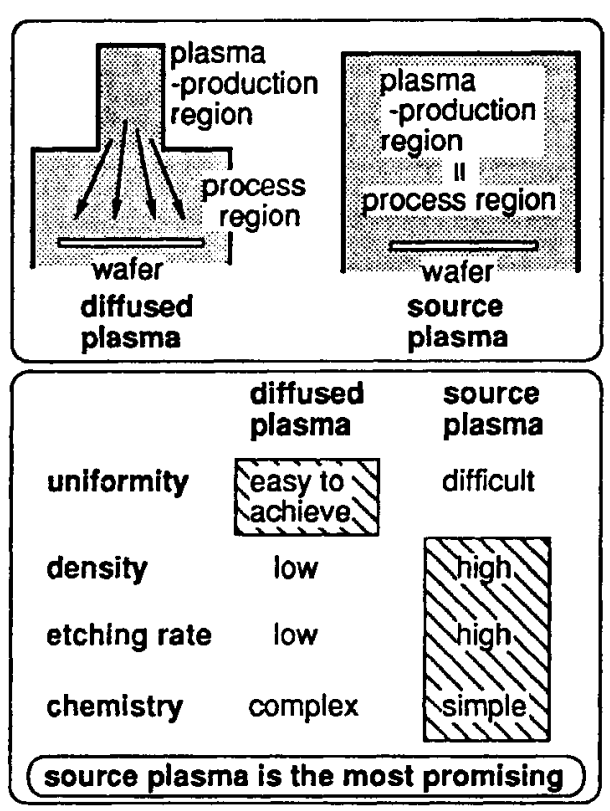

Fig. 8 Diffused plasma and source plasma. 


\subsection{Source plasma and three-dimensionally-uniform plasma}

A large plasma can be made either by using a diffused plasma or by using a source plasma, as shown in Fig. 8. With the diffused plasma, a small high-density plasma is made in the plasma-production region, and then it is diffused into the process region to make a large low-density plasma. With the source plasma, a large plasma is produced directly in the process region. Good uniformity is achieved more easily with the diffused plasma, while high-density plasma at the wafer position and a high etching rate are achieved more easily with the source plasma. The gas-phase chemistry of the two methods also has important differences. In the diffused plasma, plasma parameters, such as the electron and ion temperatures, differ significantly between the plasma-production region and the process region. But, with a source plasma, the parameters are the same throughout the vacuum chamber, so the gas-phase chemistry is simpler with the source plasma. This suggests that the source plasma offers better control of the process. In the past, the uniformity of plasma in the vicinity of the wafer surface has been examined mainly in the two-dimensional sense. However, in the near future, the development of a three-dimensionally uniform plasma is sure to become very important for the application of plasma etching to practical semiconductor-production lines.

\subsection{Importance of chamber-wall-condition control}

We have to process a large-diameter wafer uniformly in a three-dimensional sense. Also, we have to process the wafer at a high etching rate by using a small-volume plasma at a high gas-flow rate, namely by making a gas-residence time short. These requirements result in etching with a plasma that is thin and has a large diameter. When etching with such a plasma, the chamber wall is very close to the wafer surface, which affects the etching results, such as the etched shape, the etching rate, the selectivity, and contamination. Control of the chamber-wall conditions, such as the wall temperature and adsorption, will be essential in the process.

\section{Post-Plasma Etching}

The problem of damage caused in plasma etching by the charged and/or energetic particles that is an integral part of a plasma, must be overcome by post-plasma etching techniques using charge-free and/or low-energy processes. Potential techniques include neutral-beam etching (12), hot-molecular-beam etching (13), photo-assisted etching (14), and multiply-charged-ion-beam etching (15). A charge-free process is needed especially for insulators, but may not always be needed for semiconductors and metals; a lowenergy process will be required for both types of materials. Further investigation on the proposed techniques is needed to realize practical applications. In addition, the use of these techniques for special requirements, such as in atomic-layer etching and formation of nanometer-size memory bits, will become more important in the future.

\section{Summary}

The progress of dry-etching techniques in recent years was investigated by taking an overall view of the mechanism of plasma etching and the development of etching methodologies. We conclude that the primary goal of future research should be to develop new precisely controllable parameters, with the aim of improving current plasma-etching techniques. Our belief is based on the three-level structure of the parameters in plasma etching. Potential new plasmas, such as a soft three-dimensionally-uniform plasma, will help advance this research. The development of post-plasma etching techniques is also growing in importance with increasing device integration and the appearance of new device concepts. In this area, achieving improved performance with less damage is the key to more refined etching in the future.

\section{References}

1. J.W. Coburn et al. J.Appl.Phys. 50, 3189 (1979).

2. D.L. Flamm et al. J.Vac.Sci.Technol. B1, 23 (1983).

3. K. Suzuki et al. Jpn.J.Appl.Phys. 16, 1979 (1977).

4. K. Suzuki et al. J.Vac.Sci.Technol. B3, 1025 (1985).

5. S. Tachi et al. Appl.Phys.Lett. 52, 616 (1988).

6. K. Tsujimoto et al. Extended Abstracts of the 18th Conference on Solid State Devices and Materials (1986, Tokyo), p.229.

7. K. Tsujimoto et al. J.Vac.Sci.Technol. A12, 1209 (1994).

8. J.M. Cook et al. J.Vac.Sci.Technol. B8, 1 (1990).

9. R.W. Boswell et al. Appl.Phys.Lett. 47, 1095 (1985).

10. Lam Research Corporation, Technical Note TN-003, (1992).

11. J. Givens et al. J.Vac.Sci.Technol. B12, 427 (1994).

12. T. Mizutani et al. J.Vac.Sci.Technol. A6, 1417 (1988).

13. K. Suzuki et al. J.Appl.Phys. 64, 3697 (1988).

14. P.A. Maki et al. Appl.Phys.Lett. 55, 91 (1989).

15. K. Mochiji et al. Jpn.J.Appl.Phys. 33, 7108 (1994). 